Supporting Information for

\title{
Preparation of Fe/Ni Bimetallic Oxide Porous Graphene Composite Materials for Efficient Adsorption and Removal of Sulfonamides
}

\author{
Yu Wang ${ }^{\dagger, *}$, Jia Chen ${ }^{\dagger, *}$, Ming Guan ,*, Hongdeng Qiü,\&,\&,*
}

$\dagger$ CAS Key Laboratory of Chemistry of Northwestern Plant Resources and Key Laboratory for Natural Medicine of Gansu Province, Lanzhou Institute of Chemical Physics, Chinese Academy of Sciences, Lanzhou 730000, China

\$ Laboratory on Pollution Monitoring and Control, College of Chemistry and Chemical Engineering, Xinjiang Normal University, Urumqi 830054, China

$\S$ College of Chemistry, Zhengzhou University, Zhengzhou 450001, China

${ }^{\&}$ College of Chemistry and Chemical Engineering, Gannan Normal University, Ganzhou 341000, China

*Corresponding e-mail: jiachen@licp.cas.cn (J. Chen); guanm@xjnu.edu.cn (M. Guan); hdqiu@licp.cas.cn (H. Qiu) 


\section{HPLC Conditions}

The concentration of the six SAs antibiotics is determined by the Agilent 1260 Infinity series HPLC system (Agilent Technologies, USA). Chromatographic stationary phase is ZORBAX SB-C18 column (5 $\mu \mathrm{m}, 4.6$ $\mathrm{mm} \times 250 \mathrm{~mm}$ i.d.). The mobile phase components are A- $0.2 \%$ acetic acid aqueous solution, B-methanol, and the detection wavelength is $250 \mathrm{~nm}$. The gradient elution procedure is as follows: $0 \sim 3 \mathrm{~min} 10 \% \mathrm{~B} ; 3 \sim 4 \mathrm{~min}$ $10 \% \mathrm{~B} \sim 20 \% \mathrm{~B} ; 4 \mathrm{~min} \sim 8 \mathrm{~min} 20 \% \mathrm{~B} ; 8 \mathrm{~min} \sim 15 \min 20 \% \mathrm{~B} \sim 30 \% \mathrm{~B} ; 15 \mathrm{~min} \sim 17 \min 30 \% \mathrm{~B} \sim 45 \% \mathrm{~B}$, flow rate: $1 \mathrm{~mL} / \mathrm{min}$, injection volume: $10 \mu \mathrm{L}$. 

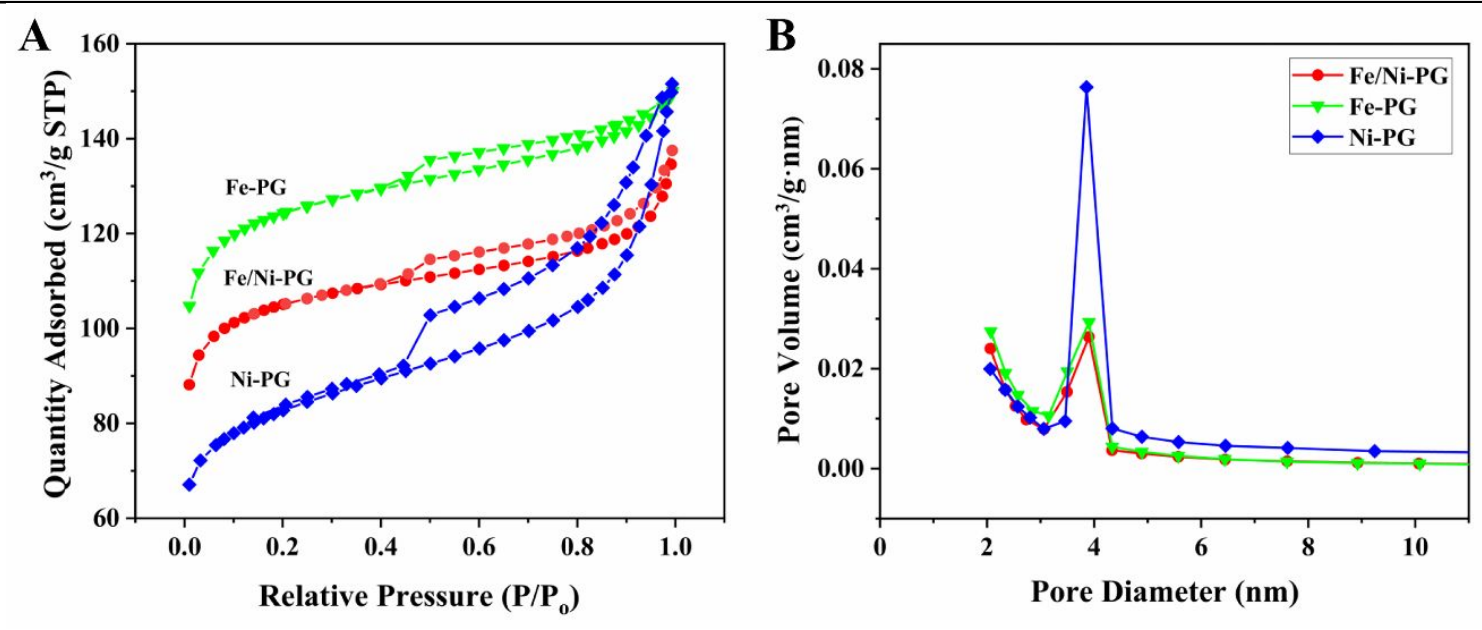

Figure S1 $\mathrm{N}_{2}$ adsorption-desorption isotherms (A) and pore size distribution (B) of Ni-PG, Fe-PG and Fe/NiPG. 

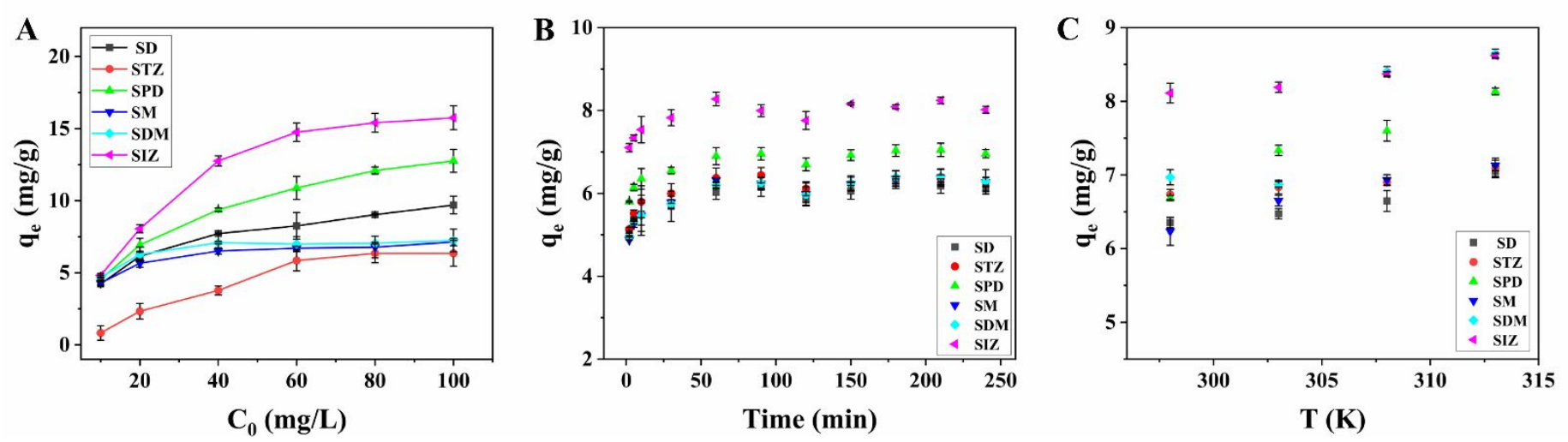

Figure S2 Effects of initial concentration(A), adsorption time(B) and temperature (C) on adsorption of SAs by $\mathrm{Fe} / \mathrm{Ni}-\mathrm{PG}$. 


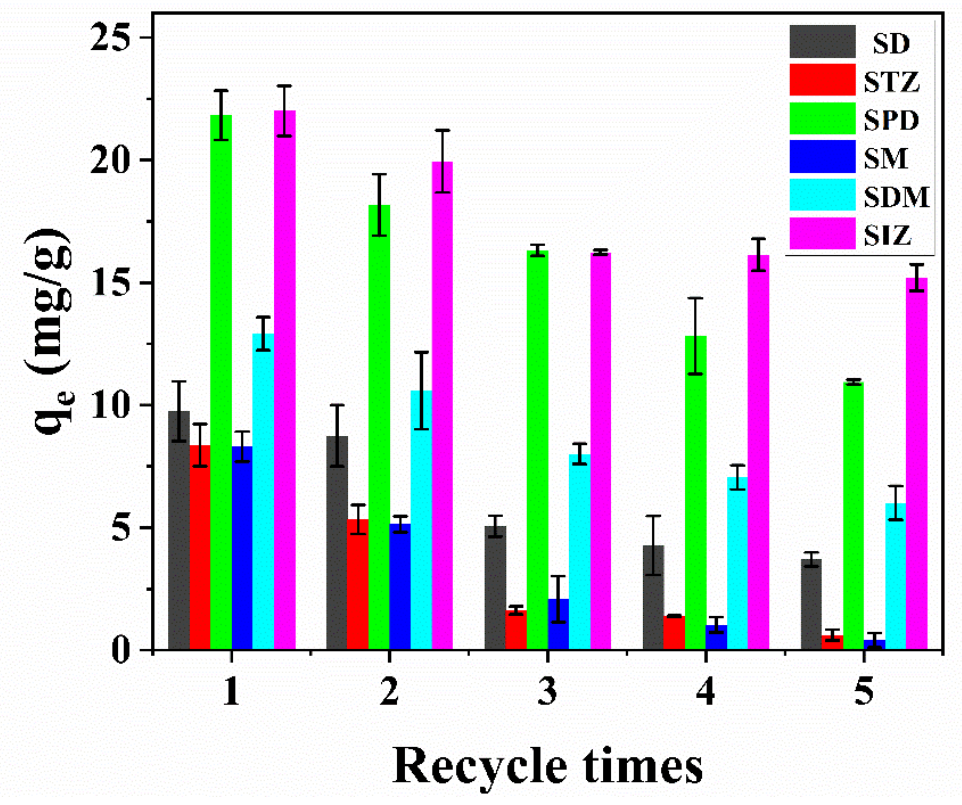

Figure S3 Reusability of the Fe/Ni-PG on adsorption of SAs. 

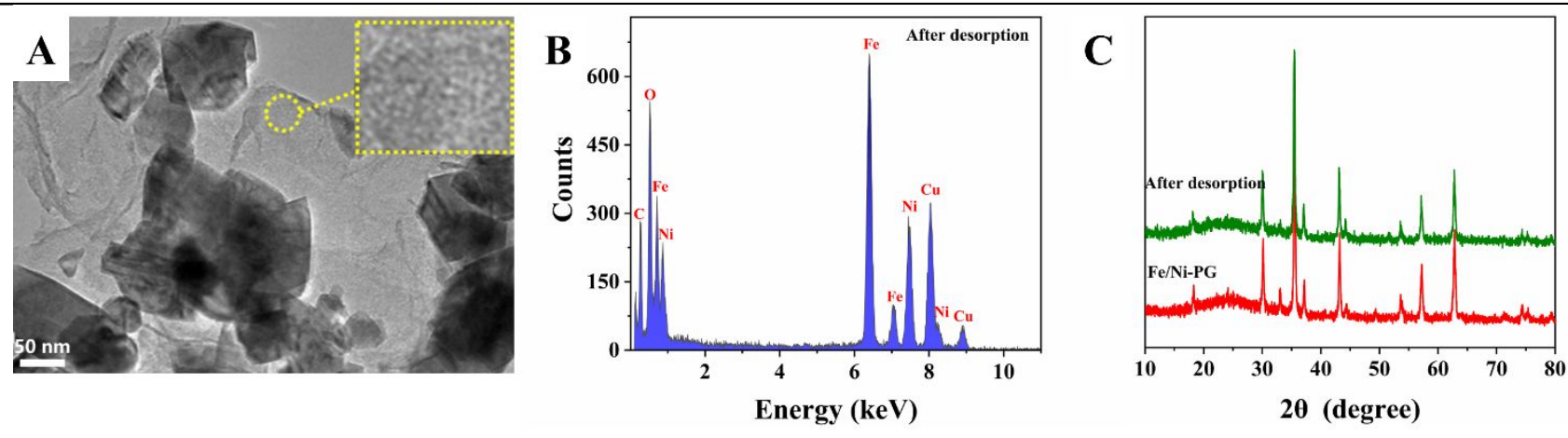

Figure S4 TEM (A) and EDX (B) of Fe/Ni-PG after desorption, XRD patterns (C) of Fe/Ni-PG and Fe/Ni-PG after desorption. 

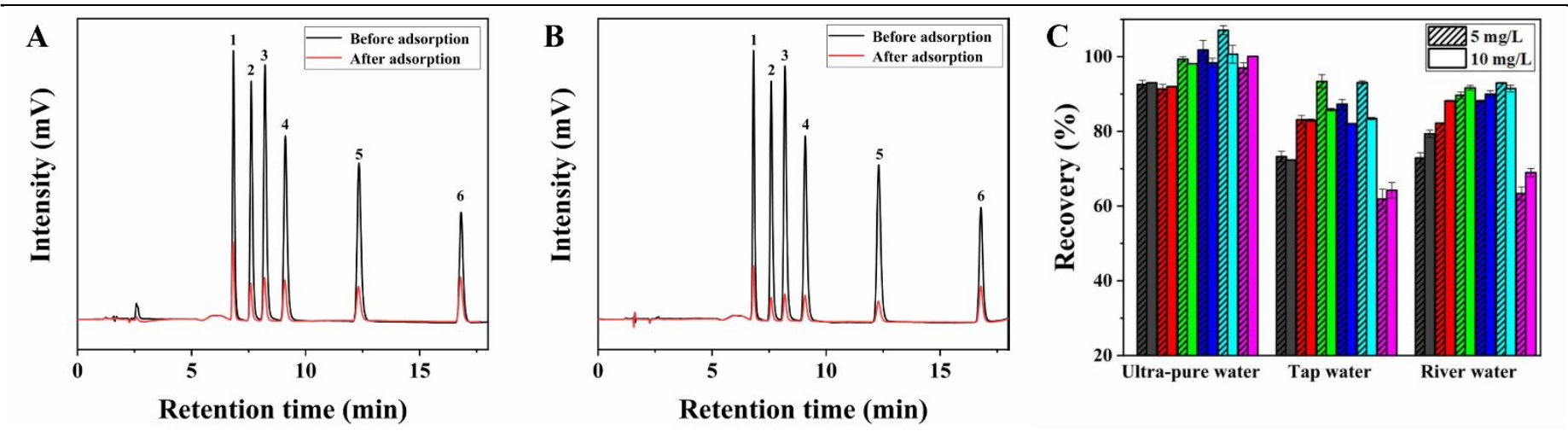

Figure S5 Chromatograms before and after the adsorption of SAs in tap water (A) and Yellow River water (B) by Fe/Ni-PG (spiked with $10 \mathrm{mg} / \mathrm{L}$ ), removal rate $(\mathrm{C})$ of $\mathrm{Fe} / \mathrm{Ni}-\mathrm{PG}$ on SAs. 
Table S1. The physiochemical properties and structures of the six SAs.

\begin{tabular}{|c|c|c|c|c|}
\hline Antibiotics & Structure & $\begin{array}{c}\text { Molecular } \\
\text { formula }\end{array}$ & $\begin{array}{l}\text { Molecular weight } \\
(\mathrm{g} / \mathrm{mol})\end{array}$ & $\mathrm{pKa}$ \\
\hline $\begin{array}{l}\text { Sulfadiazine } \\
\text { (SD) }\end{array}$ & & $\mathrm{C}_{10} \mathrm{H}_{10} \mathrm{~N}_{4} \mathrm{O}_{2} \mathrm{~S}$ & 250.28 & $\begin{array}{l}\mathrm{pKa}_{1}=1.57^{\mathrm{a}} \\
\mathrm{pKa}_{2}=6.50^{\mathrm{a}}\end{array}$ \\
\hline $\begin{array}{l}\text { Sulfathiazole } \\
\text { (STZ) }\end{array}$ & & $\mathrm{C}_{9} \mathrm{H}_{9} \mathrm{~N}_{3} \mathrm{O}_{2} \mathrm{~S}_{2}$ & 255.32 & $\begin{array}{c}\mathrm{pKa}_{1}=2.0^{\mathrm{b}} \\
\mathrm{pKa}_{2}=7.11^{\mathrm{b}}\end{array}$ \\
\hline $\begin{array}{l}\text { Sulfapyridine } \\
\text { (SPD) }\end{array}$ & & $\mathrm{C}_{11} \mathrm{H}_{11} \mathrm{~N}_{3} \mathrm{O}_{2} \mathrm{~S}$ & 249.29 & $\begin{array}{l}\mathrm{pKa}_{1}=2.30^{\mathrm{c}} \\
\mathrm{pKa}_{2}=8.40^{\mathrm{c}}\end{array}$ \\
\hline $\begin{array}{l}\text { Sulfamerazine } \\
\text { (SM) }\end{array}$ & & $\mathrm{C}_{11} \mathrm{H}_{12} \mathrm{~N}_{4} \mathrm{O}_{2} \mathrm{~S}$ & 264.30 & $\begin{array}{l}\mathrm{pKa}_{1}=2.06^{\mathrm{b}} \\
\mathrm{pKa}_{2}=6.09^{\mathrm{b}}\end{array}$ \\
\hline $\begin{array}{l}\text { Sulfamethazine } \\
\text { (SDM) }\end{array}$ & & $\mathrm{C}_{12} \mathrm{H}_{14} \mathrm{~N}_{4} \mathrm{O}_{2} \mathrm{~S}$ & 278.33 & $\begin{aligned} \mathrm{pKa}_{1} & =2.30^{\mathrm{b}} \\
\mathrm{pKa}_{2} & =7.49^{\mathrm{b}}\end{aligned}$ \\
\hline $\begin{array}{l}\text { Sulfisoxazole } \\
\text { (SIZ) }\end{array}$ & & $\mathrm{C}_{11} \mathrm{H}_{13} \mathrm{~N}_{3} \mathrm{O}_{3} \mathrm{~S}$ & 267.30 & - \\
\hline
\end{tabular}

${ }^{\mathrm{a}}$ from Ref. $1 ;{ }^{\mathrm{b}}$ from Ref. $2 ;{ }^{\mathrm{c}}$ from Ref. 3 . 
Table S2 The proportion of each element in Fe/Ni-PG.

\begin{tabular}{cc}
\hline Name & Atomic\% \\
\hline $\mathrm{C} 1 \mathrm{~s}$ & 84.1 \\
$\mathrm{Fe} 2 \mathrm{p}$ & 1.22 \\
$\mathrm{Ni2p}$ & 1.36 \\
$\mathrm{O} 1 \mathrm{~s}$ & 13.3 \\
\hline
\end{tabular}


Table S3 The BET parameters for Ni-PG, Fe-PG and Fe/Ni-PG.

\begin{tabular}{ccccc}
\hline Sample & $\begin{array}{c}\mathrm{q}_{\mathrm{m}} \\
(\mathrm{mg} / \mathrm{g})\end{array}$ & $\begin{array}{c}\mathrm{S}_{\mathrm{BET}} \\
\left(\mathrm{m}^{2} / \mathrm{g}\right)\end{array}$ & $\begin{array}{c}\text { Pore Volume } \\
\left(\mathrm{cm}^{2} / \mathrm{g}\right)\end{array}$ & $\begin{array}{c}\text { Pore Size } \\
(\mathrm{nm})\end{array}$ \\
\hline Ni-PG & 23 & 282.30 & 0.151 & 6.18 \\
Fe-PG & 58 & 421.02 & 0.077 & 3.79 \\
Fe/Ni-PG & 63 & 355.78 & 0.082 & 4.72 \\
\hline
\end{tabular}




\section{REFERENCES}

(1) Manjunath, S. V.; Singh Baghel, R.; Kumar, M., Antagonistic and synergistic analysis of antibiotic adsorption on Prosopis juliflora activated carbon in multicomponent systems. Chem. Eng. J. 2020, 381, 122713.

(2) Li, R.; Zhang, Y.; Chu, W.; Chen, Z.; Wang, J., Adsorptive removal of antibiotics from water using peanut shells from agricultural waste. $R S C A d v$. 2018, 8 (24), 13546-13555.

(3) Yao, Y.; Zhang, Y.; Gao, B.; Chen, R.; Wu, F., Removal of sulfamethoxazole (SMX) and sulfapyridine (SPY) from aqueous solutions by biochars derived from anaerobically digested bagasse. Environ. Sci. Pollut. Res. Int. 2018, 25 (26), 2565925667. 